\title{
Distribution of Partial Neutron Widths for Nuclei Close to a Maximum of the Neutron Strength Function
}

\author{
Hans A. Weidenmüller ${ }^{1}$ \\ ${ }^{1}$ Max-Planck-Institut für Kernphysik \\ Heidelberg, Germany
}

\begin{abstract}
For nuclei near a maximum of the neutron strength function, the secular dependence on energy $E$ of $s$-wave partial neutron widths differs from the canonical form $\sqrt{E}$. We derive the universal form of that dependence and show that it is expected to significantly influence the analysis of neutron resonance data.
\end{abstract}

PACS numbers: $24.30 \mathrm{Gd}, 24.60 \mathrm{Dr}, 24.60 \mathrm{Lz}, 25.40 \mathrm{Lw}$

Purpose. The Porter-Thomas distribution [1] is one of the key predictions of Random-Matrix Theory (RMT). The reduced partial neutron widths (simply "neutron widths" in the sequel) of compound-nucleus $(\mathrm{CN})$ resonances are predicted to follow a $\chi^{2}$ distribution with $\nu=1$ degrees of freedom. That prediction was recently tested with unprecedented accuracy [2]. The authors scattered slow neutrons on several Pt isotopes, thereby measuring sequences of CN resonances over energy intervals of up to $20 \mathrm{keV}$ length, and obtained sets of widths for $s$-wave neutrons containing up to 450 data points. Reduced neutron widths were obtained by rescaling the measured widths by the factor $f^{2}(E)=\sqrt{E}$ with $E$ taken at resonance energy. That factor is supposed to take account of the secular variation with energy of the widths for $s$-wave neutrons. Using a cutoff procedure to minimize $p$-wave background and a maximum-likelihood analysis, the authors concluded that the validity of the Porter-Thomas distribution must be rejected with a statistical significance of at least 99.997 per cent [2]. That result calls into question earlier successful tests of RMT in nuclei (for a review, see Ref. [3]). More generally, it questions whether at excitation energies of several $\mathrm{MeV}$ nuclei are correctly described as basically chaotic systems, a view widely held so far. Not surprisingly, the result announced by Koehler et al. has found wide attention [4].

For the isotopes of Pt investigated in Ref. [2], the neutron strength function (the ratio of the average neutron partial width and the mean resonance spacing) is strongly enhanced. The enhancement facilitates the separation of $s$-wave resonances from the contamination of small $p$-wave resonances. To the best of our knowledge, the theoretical implications of that enhancement for the secular dependence of neutron widths on energy, i.e., for the function $f^{2}(E)$ introduced above, have never been investigated. In this Letter we show that the enhancement of the strength function implies that $f^{2}(E)$ carries an additional energy dependence on top of the $\sqrt{E}$-dependence mentioned above. That dependence has never been taken into account in any analysis of data on neutron widths. It is not clear whether the conclusions drawn in Ref. [2] will be upheld when that dependence is accounted for.

Maxima of the neutron strength function are due to a resonance close to threshold (more precisely: to a virtual state as defined below) or to a weakly bound state of the nuclear single-particle potential for $s$-wave neutrons. Such maxima occur systematically with increasing mass number (i.e., increasing radius of the nuclear single-particle potential) when a bound $s$-wave state is being formed at threshold. (For the Pt isotopes that is the $4 s$-state in spectroscopic notation). The enhancement of neutron widths due to the nascent bound singleparticle state is strongest for $\mathrm{CN}$ resonances close to neutron threshold and, being a threshold effect, dies out with increasing separation of the CN resonances from neutron threshold. Such weakening of the enhancement causes the additional energy dependence of $f^{2}(E)$ and forms the topic of the present paper.

To make the paper self-contained we begin with a brief account of single-channel CN scattering theory. We show that $f(E)$ is determined by the projection of the neutron single-particle scattering wave function onto the nuclear volume. We calculate that projected part of the wave function for an attractive square-well potential and determine $f(E)$ for both, a single-particle resonance close to threshold and a weakly bound state. We show that the enhancement is a generic feature and occurs likewise in more realistic nuclear single-particle potentials that differ from a square well. Whenever the $s$-wave strength function shows significant enhancement, $f^{2}(E)$ differs significantly from $\sqrt{E}$ even over energy intervals as small as $20 \mathrm{keV}$ (the interval used in Ref. [2]).

Single-Channel CN Scattering. We deal with a single channel (the $s$-wave neutron channel). The energy $E$ is positive and $E=0$ denotes the threshold. In the shellmodel approach to nuclear reactions [5], the channel wave function $\chi_{E}$ is the antisymmetrized product of the wave function of the target nucleus in its ground state and the neutron single-particle scattering wave function $\Psi_{E}(r)$. The latter depends only on the radial coordinate $r$ and is chosen real. The numerous many-body resonances typical for CN scattering are due to $N \gg 1$ orthonormal quasibound states $|\mu\rangle$ where $\mu=1,2, \ldots, N$. These in- 
teract with each other through the $N$-dimensional real and symmetric Hamiltonian matrix $\langle\mu|H| \nu\rangle=H_{\mu \nu}$ and are coupled to the neutron channel by real Hamiltonian matrix elements

$$
W_{\mu}(E)=\left\langle\chi_{E}|H| \mu\right\rangle .
$$

The unitary scattering amplitude $S(E)$ that takes into account the presence of the $N \mathrm{CN}$ resonances has the form $[\underline{5},[6]$

$$
S(E)=\exp [2 i \delta]\left(1-2 i \pi \sum_{\mu, \nu=1}^{N} W_{\mu}(E) D_{\mu \nu}^{-1}(E) W_{\nu}(E)\right) .
$$

Here $\delta$ is the $s$-wave potential scattering phase shift, and

$$
D_{\mu \nu}(E)=E \delta_{\mu \nu}-H_{\mu \nu}-F_{\mu \nu}(E)+i \pi W_{\mu}(E) W_{\nu}(E)
$$

where

$$
F_{\mu \nu}(E)=\int_{0}^{\infty} \mathrm{d} E^{\prime} \frac{\mathcal{P}}{E-E^{\prime}} W_{\mu}\left(E^{\prime}\right) W_{\nu}\left(E^{\prime}\right)
$$

and where $\mathcal{P}$ denotes the principal-value integral. The last term in Eq. (3) is proportional to the width matrix and descibes the instability of the $\mathrm{CN}$ resonances due to their coupling to the channel. The matrix $F_{\mu \nu}(E)$ in Eq. (4) accounts for the shift of the resonances due to that coupling. Eqs. (2) to (4) provide a very general framework for $s$-wave neutron scattering in the presence of $\mathrm{CN}$ resonances. The assumption that the $\mathrm{CN}$ resonances are described by RMT is implemented by postulating that the matrix $H_{\mu \nu}$ is a member of the Gaussian Orthogonal Ensemble (GOE) of random matrices.

Near neutron threshold the $\mathrm{CN}$ resonances are isolated, and we use the diagonal representation $H_{\mu \nu}=$ $\sum_{\rho} \mathcal{O}_{\rho \mu} E_{\rho} \mathcal{O}_{\rho \nu}$ where $\mathcal{O}_{\mu \nu}$ is orthogonal and where $E_{\rho}$ are the eigenvalues of $H_{\mu \nu}$. We define $\tilde{W}_{\mu}(E)=\sum_{\nu} \mathcal{O}_{\mu \nu} W_{\nu}$ and $\tilde{F}$ by Eq. (4) with all $W$ s replaced by $\tilde{W}_{\mathrm{s}}$. For isolated resonances the coupling between the eigenvalues $E_{\rho}$ is negligible (both $\tilde{F}_{\mu \nu}$ and $\tilde{W}_{\mu} \tilde{W}_{\nu}$ are taken to be diagonal), and $S(E)$ takes the form

$$
S(E)=\exp [2 i \delta]\left(1-2 i \pi \sum_{\mu} \tilde{W}_{\mu}(E)\left(E-\mathcal{E}_{\mu}\right)^{-1} \tilde{W}_{\mu}(E)\right)
$$

where the complex resonance energies $\mathcal{E}_{\mu}$ are given by

$$
\mathcal{E}_{\mu}=E_{\mu}+\tilde{F}_{\mu \mu}-i \pi \tilde{W}_{\mu}^{2} .
$$

The neutron partial width amplitude of the resonance located at $\mathcal{E}_{\mu}$ is given by $\sqrt{2 \pi} \tilde{W}_{\mu}(E)=$ $\sqrt{2 \pi} \sum_{\nu} \mathcal{O}_{\mu \nu} W_{\mu}(E)$. In order to remove any secular energy dependence from the matrix elements $W_{\mu}(E)$ we write

$$
W_{\mu}(E)=f(E) V_{\mu} .
$$

By construction, the amplitudes $V_{\mu}$ do not carry any secular dependence on energy $E$. GOE predicts that in the limit of infinite matrix dimension, the elements $\mathcal{O}_{\mu \nu}$ and, therefore, the amplitudes $\sum_{\nu} \mathcal{O}_{\mu \nu} V_{\nu}$ are Gaussiandistributed random variables. As a consequence, the reduced neutron widths $2 \pi \tilde{W}_{\mu}^{2}(E) / f^{2}(E)$ are predicted to follow the Porter-Thomas distribution. To test that prediction, we must determine the function $f(E)$ for nuclei in the vicinity of a single-particle $s$-wave resonance close to threshold and for a weakly bound single-particle $s$ wave state.

Poles of the Single-Particle Scattering Amplitude. Eq. (11) shows that the entire energy dependence of $W_{\mu}(E)$ is due to $\chi_{E}$. We recall that $\chi_{E}$ is the antisymmetrized product of the ground-state wave function of the target nucleus and the real $s$-wave scattering wave function $\Psi_{E}(r)$. For the matrix element $W_{\mu}(E)$, only the projection of $\Psi_{E}$ onto the nuclear volume is relevant. Thus, the energy dependence of $W_{\mu}(E)$ is determined by the enery dependence of $\Psi_{E}(r)$ for $r \leq R$ where $R$ is the nuclear radius.

The scale in energy over which the radial dependence of a single-particle scattering wave function changes, is given by the typical distance between bound $s$-wave single-particle states. In a heavy nucleus, that distance is about $10 \mathrm{MeV}$ and, thus, very large compared to the typical energy scale over which resonance data are taken. (In Ref. 2], that scale was $20 \mathrm{keV}$ ). Therefore, the radial form of the neutron $s$-wave scattering function in the nuclear volume $r \leq R$ can safely be taken as independent of energy, and we focus attention on the energy-dependent amplitude $f(E)$ of that function. In the vicinity of the threshold energy $(E=0), f(E)$ is strongly enhanced whenever a single-particle $s$-wave resonance or a bound $s$-wave single-particle state occurs close to threshold. Both resonance and bound state manifest themselves as poles of the unitary single-particle potential scattering amplitude $s(E)=\exp [2 i \delta]$ in Eq. (2). To understand qualitatively what happens we recall some properties of the poles of $s(E)$. The potential scattering wave function $\Psi_{E}(r)$ at energy $E$ depends asymptotically on wave number $k$ where $\hbar^{2} k^{2} /(2 m)=E$, and it is useful to consider the distribution of poles of the scattering amplitude $s(E)$ in the complex $k$-plane (rather than the complex energy plane). We accordingly replace $s(E)$ by $s(k)$.

For a square-well potential, poles of $s(k)$ have been studied in detail [5, 7]. Poles of $s(k)$ occur either pairwise or as single poles. Pairs of poles lie below the real $k$-axis and occur symmetrically to the imaginary $k$-axis. Such pairs are, thus, located at $k_{0}$ and at $-k_{0}^{*}$ with $\Im\left(k_{0}\right) \leq-i / a$ (where $a$ is the radius of the squarewell potential) and $\Re\left(k_{0}\right)>0$. Single poles lie on the imaginary $k$-axis. Poles on the positive imaginary $k$-axis correspond to bound states while poles on the negative imaginary $k$-axis are referred to as virtual states. We visualize the motion of the poles in the complex $k$-plane 
as the depth $V_{0}$ of the square-well potential is increased. For a very shallow potential, there are no bound states and no poles on the imaginary $k$-axis. All poles lie far below the real $k$-axis, and significant resonant behavior is absent. As $V_{0}$ is increased, the first pair of poles approaches the negative imaginary axis from opposite sides. The two poles coalesce at $k=-i / a$. Then one pole moves up and the other pole moves down the imaginary $k$-axis. Significant resonance behavior of $s(k)$ is caused only by the upward-moving pole, first as a virtual state and later as a weakly bound state. (For $a=6 \mathrm{fm}$, a realistic value for the radius of a heavy nucleus, the point $k=-i / a$ has a distance in energy of about $0.5 \mathrm{MeV}$ from threshold). As $V_{0}$ is increased further, resonance enhancement subsides. The pattern repeats itself as the next pair of poles approaches the point $k=-i / a$ and the $2 s$-state is pulled into the potential, and so on.

Enhancement factor for the square-well potential. We work out the resulting enhancement of the amplitude $f(E)$ for the $4 s$-state although the analysis and result are exactly the same for any $s$-state near threshold. The real scattering function $\Psi_{E}(r)$ is normalized to a delta function in energy. An elementary calculation shows that for $r<a$ we have $\Psi_{E}(r)=f(E) \sin (\kappa r) / r$ where $\hbar^{2} \kappa^{2} /(2 m)=E+V_{0}$ and where

$$
f(E)=\sqrt{\frac{m}{\pi k \hbar^{2}}} \frac{2(k a)}{\sqrt{(k a)^{2} \sin ^{2}(k a)+(\kappa a)^{2} \cos ^{2}(\kappa a)}} .
$$

As remarked above, the function $\sin (\kappa r)$ changes very slowly with energy, and attention is focused on $f(E)$.

A weakly bound $4 s$-state with energy binding $E_{0}<0$ exists if the condition $[(k a) /(\kappa a)] \tan (\kappa a)=-1$ is met for $(\kappa a)=(7 \pi / 2)+\varepsilon$ and $\varepsilon \ll 1$. Then $E_{0}=$ $-(7 \pi / 2)^{2}\left(\hbar^{2} \varepsilon^{2}\right) /\left(2 m a^{2}\right)$. Expanding $(\kappa a), \sin (\kappa a)$ and $\cos (\kappa a)$ in Taylor series around $\kappa a=7 \pi / 2$ and keeping only lowest-order terms, we find

$$
f^{2}(E) \propto \frac{\sqrt{E}}{E+\left|E_{0}\right|} .
$$

We have suppressed energy-independent factors. The factor $\sqrt{E}$ is universal for $s$-wave scattering near threshold. The factor $\left(E+\left|E_{0}\right|\right)^{-1}$ describes the enhancement due to the weakly bound single-particle $s^{-}$ wave state. For the virtual state the condition reads $[(k a) /(\kappa a)] \tan (\kappa a)=+1$. That yields for $f^{2}(E)$ the same form as in Eq. (9) except that now $E_{0}$ is the energy associated with the virtual state on the negative imaginary $k$-axis.

We have expanded $(\kappa a)$ in powers of $(k a)$ around the location of the pole of $s(E)$, and we have kept only the term of zeroth order. The term of next order is $(k a)^{2} /(7 \pi)$. For $0 \leq E \leq 100 \mathrm{keV}$ and $a=6 \mathrm{fm}$, that term is not larger than 0.01 , and we expect the pole approximation in Eq. (9) to be excellent.
Discussion. Although derived specifically for a squarewell potential, the factor given in Eq. (9) is universal and describes for $r \leq R$ the enhancement of the square of the $s$-wave single-particle scattering wave function also for other, more realistic forms of the single-particle potential. Indeed, the pattern of movement of the poles of $s(k)$ in the complex $k$-plane versus depth of the potential is the same for all potentials that lack a barrier. Pairs of $s$-wave poles occur some distance below the real $k$-axis and symmetrically to the imaginary axis and cannot give rise to significant resonance enhancement. Therefore, the value of $k$ where such pairs coalesce on the negative imaginary $k$-axis, although different from that of the squarewell potential, amounts to at least several $100 \mathrm{keV}$. Significant resonance behavior is again due to the pole of $s(k)$ that moves up on the imaginary $k$-axis from the point of coalescence, first as a virtual and then as a weakly bound state. Potential scattering theory [5] shows that at a pole of $s(k), \Psi_{E}(r)$ is singular. For $k$ positive and close to a pole on the imaginary $k$-axis, $f^{2}(E)$ has the form of Eq. (91).

The universality of our result is displayed by the fact that $f^{2}(E)$ in Eq. (9) depends only on the energy of the virtual or weakly bound state and is independent of any detailed properties of the potential. (The value of $E_{0}$ in Eq. (9) does, of course, depend on the potential). The denominator in Eq. (9) is obviously a special case of the universal Lorentzian factor $\left[\left(E-E_{0}\right)^{2}+(1 / 4) \Gamma^{2}\right]^{-1 / 2}$ describing resonance enhancement and applies when the resonance is located below threshold so that $E_{0}$ is negative and $\Gamma$ vanishes. We also note that the enhancement factor in Eq. (9) is similar to that due to a doorway state.

We conclude that for a virtual or a weakly bound neutron $s$-wave state, the partial neutron widths carry the universal enhancement factor given in Eq. (9). The enhancement factor affects both, the neutron strength function and the secular energy dependence of the widths of neutron $s$-wave resonances. To see what happens to the neutron strength function, we keep $E$ (or $k$ ) fixed, positive, and slightly above threshold, and we increase mass number $A$, thereby increasing the radius of the potential. A pair of $s$-wave neutron resonances approaches the imaginary $k$-axis, eventually giving rise to a virtual and, later, to a bound state. For the virtual state, the energy $\left|E_{0}\right|$ decreases monotonically toward zero and then, as the virtual state turns into a bound state, increases monotonically from zero. The result is first a steady increase of the enhancement factor (9) and then, after the virtual state becomes a bound state, a decrease of that factor. Taken together, that causes the characteristic maximum of the strength function. Conversely, when neutron widths are measured near threshold for a sequence of $s$-wave resonances at some fixed value of $A$ where the strength function is enhanced and, therefore, the enhancement factor in Eq. (9) is operative, the resulting secular energy dependence of the neutron widths 
goes beyond the standard $\sqrt{E}$-dependence.

Given the universality of the enhancement factor in Eq. (9), we ask how that factor is expected to affect the analysis of neutron resonance data. We take the work of Ref. 2] as an example. We assume that the distance of $\left|E_{0}\right|$ from threshold is large compared to the average resonance spacing. We recall that the data typically range over an energy interval of $20 \mathrm{keV}$. To predict the influence of the enhancement factor in Eq. (9) on the data, one would have to determine $E_{0}$ with an accuracy of about $100 \mathrm{keV}$. That requirement precludes a theoretical prediction using the neutron single-particle potential in heavy nuclei. That potential is not known to such an accuracy. Therefore, we use another estimate. The mass dependence of single-particle levels is governed by the factor $A^{-2 / 3}$. For a potential depth of several ten $\mathrm{MeV}$ and $A \approx 200$, a weakly bound single-particle level changes by about $150 \mathrm{keV}$ when $A$ changes by one unit. Therefore, we expect that for nuclei near the maximum of the neutron strength function, $\left|E_{0}\right|$ is of the order of $100 \mathrm{keV}$. That is also consistent with the fact that for the square-well potential, the point of coalscence has a distance of about $500 \mathrm{keV}$ from threshold. Taking in Eq. (9) $\left|E_{0}\right|=50 \mathrm{keV}(10 \mathrm{keV})$ as examples, we see that the resonance enhancement factor (the denominator of expression (9)) changes by a factor 1.4 (a factor 3 , respectively) over an interval of $20 \mathrm{keV}$ starting from threshold. Thus, the resonance enhancement in Eq. (9) may significantly influence the analysis of data on neutron widths.

In such an analysis, a first estimate of $E_{0}$ can be obtained from Eq. (9) and the measured enhancement of the strength function. One may then consider $E_{0}$ and the average value of the reduced neutron partial widths as free parameters, and search for a best fit to a $\chi^{2}$ distribution with $\nu$ degrees of freedom for the reduced partial widths. Alternatively, it is possible to determine the enhancement factor directly, i.e., without using the pole approximation of Eq. (9). That can be done by solving numerically the radial Schrödinger equation for $s$-wave neutrons for a potential that is realistic in form and that possesses a virtual or a bound state close to threshold. The factor $f(E)$ is then determined by the delta-function normalization condition for the solution of the radial equation. We stress that the single-particle nuclear potential to be used is not the optical-model potential (which describes the average neutron scattering amplitude and, thus, incorporates the effect of the $\mathrm{CN}$ resonances) nor is it the real part of the optical-model potential (because the imaginary part contributes to the real part via a dispersion relation). Rather, it is the pure shell-model potential for neutrons. Conclusions about the failure of RMT can be drawn only if such an approach yields a value for $\nu$ that is significantly different from unity. Conversely, if agreement is obtained for $\nu \approx 1$, that should make it possible to determine the energy $E_{0}$ fairly precisely (with an error of perhaps not more than $100 \mathrm{keV}$ ) and, from there, the shell-model potential for neutrons in heavy nuclei with great accuracy.

We mention in passing that the resonance enhancement in Eq. (9) also affects the shift matrix $F_{\mu \nu}(E)$ in Eq. (4). With the same approximations as used above, the level shift $\tilde{F}_{\mu \mu}$ in Eq. (6) is given by the product of the reduced partial width for the resonance at $\mathcal{E}_{\mu}$ and the factor $\int_{0}^{\infty} \mathrm{d} E^{\prime} \mathcal{P} f^{2}\left(E^{\prime}\right) /\left(E_{\mu}-E^{\prime}\right)$. The enhancement of the shift due to the pole of $f^{2}(E)$ in Eq. (9) may be considerable and have important implications for the analysis of spectral fluctuations.

So far we have considered the case where $\left|E_{0}\right|$ is much larger than the mean spacing of the resonances. That is probably the typical case. If that condition fails (i.e., if $\left|E_{0}\right| \leq 5 \mathrm{keV}$ or so), it is not justified to consider the neutron widths as energy-independent constants. Then it is essential to display the full energy dependence of $S(E)$ explicitly. We use Eqs. (7) and (9) and rewrite the last term in Eq. (5) as

$-2 i \pi \sum_{\mu} \sqrt{E} \tilde{V}_{\mu}\left[\left(E+\left|E_{0}\right|\right)\left(E-E_{\mu}-\tilde{F}_{\mu \mu}\right)+i \pi \sqrt{E} \tilde{V}_{\mu}^{2}\right]^{-1} \tilde{V}_{\mu}$.

Here $\tilde{V}_{\mu}=\sum_{\nu} \mathcal{O}_{\mu \nu} V_{\nu}$. Eq. (10) is qualitatively different from Eq. (5) with energy-independent widths. The formulas of $R$-matrix theory commonly used for the analysis of neutron cross-section data bear a close analogy to the latter equation [5]. Eq. (10) shows that these formulas cannot be used when $E_{0}$ is very close to threshold.

In summary, we have shown that a substantial enhancement of the neutron strength function necessarily implies a significant energy dependence of the neutron partial widths for resonances in the vicinity of neutron threshold. We have derived the universal form of that energy dependence. Conclusions about the validity of RMT predictions can reliably be drawn only when that dependence is taken into account in the analysis of neutron resonance data.

Acknowledgments. I am grateful to E. Reich for drawing my attention to the work of Koehler et al. and to O. Bohigas, G. E. Mitchell, P. E. Koehler, and F. Becvar for stimulating correspondence.

[1] C. E. Porter and R. G. Thomas, Phys. Rev. 104, 483 (1956).

[2] P. E. Koehler, F. Becvar, M. Krticka, J. A. Harvey, and K. H. Guber, Phys. Rev. Lett. 105, 072502 (2010).

[3] H. A. Weidenmüller and G. E. Mitchell, Rev. Mod. Phys. 81, 539 (2009).

[4] E. S. Reich, Nature 466, 1034 (2010).

[5] C. Mahaux and H. A. Weidenmüller, Shell-Model Approach to Nuclear Reactions, North-Holland, Amsterdam (1969).

[6] G. E. Mitchell, A. Richter, and H. A. Weidenmüller, arXiv:1001.2422 and Rev. Mod. Phys. (in press). 
[7] H. M. Nussenzweig, Nucl. Phys. 11, 499 (1959). 